\title{
Tree Shrew Umbilical Cord Mesenchymal Stem Cells Labeled with the Dark Red Fluorescent Dye DIR and Small Animal Live Imager Observation
}

Guang-ping Ruan, Xiang Yao, Kai Wang, Jie He, Rong-qing Pang, Xiang-qing Zhu, Xing-hua Pan

10.18805/IJAR.BF-1426

\begin{abstract}
Background: Umbilical cord mesenchymal stem cell transplantation can treat metabolic syndrome, but the tracing of cells in the body after transplantation has always been a problem. Tree shrew umbilical cord mesenchymal stem cells were labeled with the dark red fluorescent dye DIR and a metabolic syndrome model in tree shrew was generated. The migration, distribution, colonization and survival of the cells were observed.

Methods: Tree shrew umbilical cord mesenchymal stem cells were labeled with the dark red fluorescent dye DIR. Three days after the tree shrew model was generated, the pancreas, kidney and liver were placed in a small animal live imager to observe the distribution of the labeled cells.

Result: The labeled cells showed deep red fluorescence in the live imager. After treatment with the transplanted cells, dark red fluorescent signals were observed in the liver, kidney and pancreas of the tree shrews but not in the untreated group and no dark red fluorescent signal was observed in the cell distribution.

Key words: DIR, Fluorescent dyes, Live animal imager, Tree shrew, Umbilical cord mesenchymal stem cells.
\end{abstract}

\section{INTRODUCTION}

DiR iodide is a family of lipophilic fluorescent dyes that can be used to stain cell membranes and other fat-soluble biological structures. The fluorescence color distinction is obvious: Dil shows orange fluorescence, $\mathrm{DiO}$ shows green fluorescence, DiD shows red fluorescence and DiR shows dark red fluorescence, which allows them to be used for multicolor imaging and flow analysis of living cells. The infrared fluorescence of DiR can penetrate cells and tissues and be used for tracking in in vivo imaging (Berninger et al. 2017).

DIR is a lipophilic fluorescent dye that can be used to stain cell membranes and other fat-soluble biological structures. The emission wavelength of DIR is invisible to the naked eye. After cell staining, DIR emits deep red fluorescence under excitation at a wavelength of $748 \mathrm{~nm}$; this dye can diffuse across the cell membrane, penetrate cells and tissues and be used for tracking in vivo imaging (Berninger et al. 2017).

Tree shrew umbilical cord mesenchymal stem cell (UCMSC) transplantation can be used to treat the tree shrew model of metabolic syndrome, but after cell transplantation, the migration, distribution, colonization and survival of the tree shrew cells need to be assessed by a good tracking method (Qiao et al. 2017). We used the dark red fluorescent dye DIR to label tree shrew UC-MSCs so that after cell transplantation, the distribution and colonization of labeled cells could be observed with a small animal in vivo imaging instrument (Cui et al. 2017), which provides a simple reference for the treatment of animal models with UC-MSCs (Dhanju et al. 2009).
The Basic Medical Laboratory of $920^{\text {th }}$ Hospital of Joint Logistics Support Force of PLA, The Integrated Engineering Laboratory of Cell Biological Medicine of State and Regions, The Transfer Medicine Key Laboratory of Cell Therapy Technology of Yunnan Province (Kunming, 650032, China).

Corresponding Author: Guang-ping Ruan and Xing-hua Pan, The Basic Medical Laboratory of $920^{\text {th }}$ Hospital of Joint Logistics Support Force of PLA, The Integrated Engineering Laboratory of Cell Biological Medicine of State and Regions, The Transfer Medicine Key Laboratory of Cell Therapy Technology of Yunnan Province (Kunming, Yunnan, 650032, China). Email: ruangp@126.com; xinghuapan@aliyun.com

How to cite this article: Ruan, G.P., Yao, X., Wang, K., He, J., Pang, R.Q., Zhu, X.Q. and Pan, X.H. (2021). Tree Shrew Umbilical Cord Mesenchymal Stem Cells Labeled with the Dark Red Fluorescent Dye DIR and Small Animal Live Imager Observation. Indian Journal of Animal Research. DOI: 10.18805/IJAR.BF-1426. Submitted: 14-08-2021 Accepted: 26-10-2021 Online: 19-11-2021

\section{MATERIALS AND METHODS}

DiR marking and identification

The work was carried out in the Basic Medical Laboratory of the $920^{\text {th }}$ Hospital of the Joint Logistics Support Force of PLA in 2019.

A $3 \mathrm{mM}$ DiR storage solution was prepared at a concentration of $10 \mathrm{mg} \mathrm{DiR}$ plus $3.3 \mathrm{ml}$ of DMSO for use. We used fourth-generation tree shrew umbilical cord mesenchymal stem cells, digested and dispersed the cells with $0.25 \%$ trypsin, terminated the reaction with $20 \%$ FBS- 
containing complete medium, pipetted the medium evenly, sucked the sample into a $10 \mathrm{ml}$ centrifuge tube and counted the cells and adjusted the cell concentration to $1 \times 106$ cells/ $\mathrm{ml}$. According to the concentration $1 \times 106$ cells $/ \mathrm{ml}$, a $1 \mathrm{ml}$ cell suspension was added to $5 \mu$ of $3 \mathrm{mM}$ DiR storage solution, incubated at $37^{\circ} \mathrm{C}$ for $10 \mathrm{~min}$ and then washed 3 times with prewarmed serum-free medium and centrifuged at $1500 \mathrm{r} / \mathrm{min}$ for $5 \mathrm{~min}$.

\section{Identification}

One hundred microliters of cell suspension (containing $1 \times 105$ cells) was placed into the plate and placed in the in vivo imaging device to observe deep red fluorescence.

\section{DIR-labeled UC-MSC distribution imaging in tree shrews}

Three days after cell transplantation, 2 tree shrews in the treatment group and 1 tree shrew in the model control group were sacrificed via the spinal cord method. The abdominal cavity was opened and the pancreas, kidneys and livers were quickly removed and fixed with $4 \%$ formaldehyde solution. The samples were placed in a small animal live imaging instrument under excitation at $748 \mathrm{~nm}$ wavelength and the distribution of the deep red fluorescence signal was observed.

\section{RESULTS AND DISCUSSION DiR marking of UC-MSCs}

In the in vivo imager, cells emitting deep red fluorescent signals were detected (Fig $1 \mathrm{~A}$ ), while unlabeled cells had no deep red fluorescent signals (Fig 1B).

\section{DIR-labeled UC-MSC in vivo distribution imaging}

After 3 days of DIR-labeled UC-MSC transplantation, the liver, pancreas and kidneys of the tree shrews in the model group and the treatment group were placed in a small animal in vivo imaging instrument. Deep red fluorescence was visible in the liver, kidney and pancreatic tissues of the tree shrews in the treatment group. The signals were observed (Fig 2A and B). Among them, UC-MSCs transplanted via the tail vein had the largest area of deep red fluorescent signal in the liver, suggesting that most of the umbilical cord MSCs transplanted via the tail vein may show chemotaxis and colonize the liver; however, no cells emitted deep red fluorescent signals in the organs of the tree shrews of the transplanted model group (Fig 2C).

Umbilical cord mesenchymal stem cells (UC-MSCs) have a high differentiation potential and can differentiate into multiple lineages (Fahmy et al. 2017). These cells have broad clinical application prospects in tissue engineering, such as bone, cartilage, muscle, tendon, ligament, nerve, liver, endothelium and myocardium (Wang et al. 2017). MSCs have been isolated from the human umbilical cord and their cell content and proliferative ability are better than those of bone marrow mesenchymal stem cells (BMSCs) (Bansal et al. 2014; Rahman et al. 2015). They have lower immunogenicity than bone marrow MSCs and have the advantages of convenient materials and no ethical controversy. Therefore, these cells have attracted more attention from researchers (Wu et al. 2017). UC-MSCs have been increasingly used to replace BMSCs due to their low immunogenicity and wide range of sources. Therefore, in this experiment, the adherent method was used to isolate and culture UC-MSCs from tree shrews and to identify and label them in vitro.

The nonprimate tree shrew, which is closer to humans in biological evolutionary history, was used in medical experiments as early as the 1970s and 1980s (Chen et al. 2017). In recent years, research on this species as an animal model for human diseases has also received increasing attention (Jiang et al. 2017) because the biological characteristics of tree shrews, such as physiology, biochemistry and anatomy, are similar to those of humans. Researchers have used tree shrews to establish animal models of various human viral diseases and bacterial infectious diseases, respiratory system diseases, endocrine diseases, nervous system diseases and tumors. Extensive research and applications have been published (Feng et al. 2017; Tong et al. 2017; Xiao et al. 2017). In this experiment,
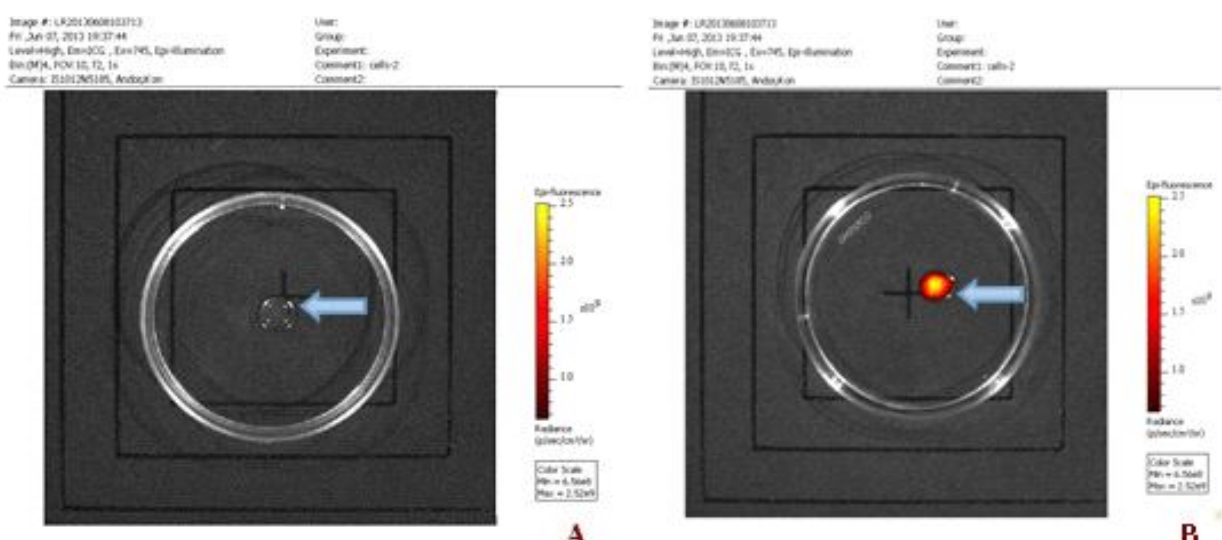

Fig 1: DiR labeling of UC-MSCs.

Note: A: unlabeled fourth-generation UC-MSCs; B: DiR-labeled fourth-generation UC-MSCs. 


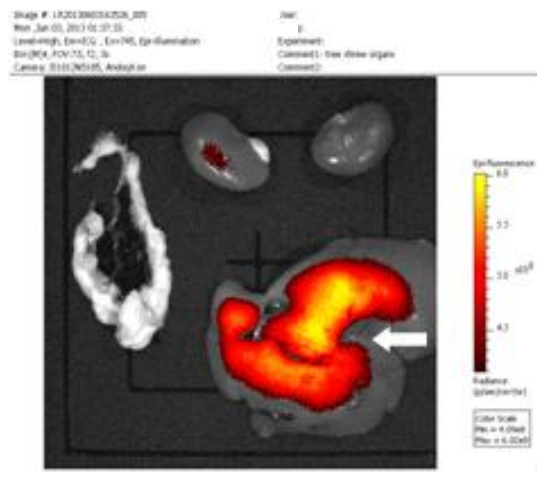

A

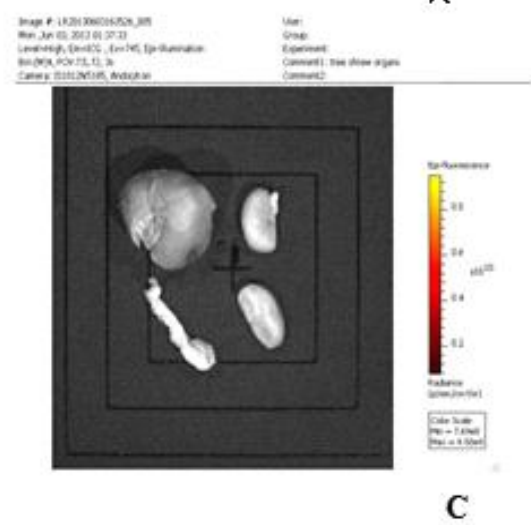

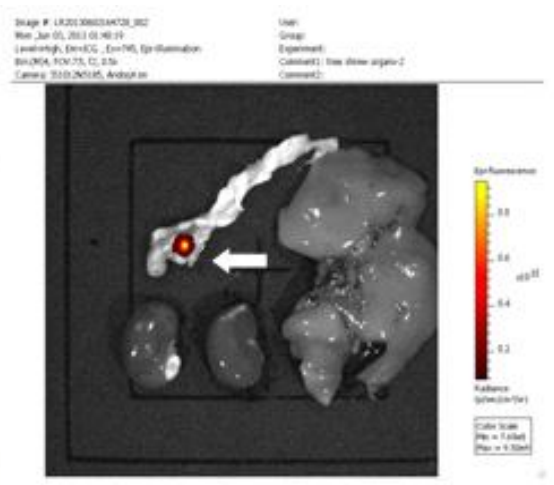

B

Fig 2: DIR staining of UC-MSCs in tree shrews of each group.

Note: A, B: liver, kidney and pancreas in the treatment group; C: liver, kidney and pancreas in the model group.

a high-sugar, high-cholesterol, high-salt diet and sugar water were used to simulate the unhealthy diet of the contemporary population and combined with streptozotocin (STZ) to induce a tree shrew metabolic syndrome model.

In this experiment, we first established a tree shrew metabolic syndrome model and then treated the model with umbilical cord mesenchymal stem cells labeled with the deep red fluorescent dye DIR.

Three days after transplantation of DIR-labeled UCMSCs, tree shrews transplanted with UC-MSCs (DIRlabeled) via the tail vein showed cell colonization with deep red fluorescent signals in the liver, kidney and pancreas; however, in the liver, the dark red fluorescent signal area was larger and the signal was stronger, indicating that most of the UC-MSCs transplanted through the tail vein may first reach and colonize the liver after passing through the blood circulation.

\section{CONCLUSION}

We used DIR-labeled cells for transplantation through the tail vein for 3 days and found that the liver showed the greatest colonization. This finding indicates that stem cells first reach and colonize the liver after entering the body through the blood circulation and then use other mechanisms to play their role in the tree shrew animal model. This issue requires more animal experiments to verify the therapeutic effects.

\section{ACKNOWLEDGEMENT}

We thank American Journal Experts for assisting with the English preparation of this manuscript.

\section{Author contribution}

Jie He, Guang-ping Ruan and Xiang Yao made substantial contributions to the study conception and design, data acquisition, or data analysis and interpretation. Jie He, Guang-ping Ruan, Kai Wang and Xiang Yao conducted the experiments. Xiang Yao and Jie He agree to be accountable for all aspects of the work and ensure that questions related to the accuracy or integrity of any part of the study will be appropriately investigated and resolved. Xing-hua Pan and Guang-ping Ruan provided final approval of this version of the manuscript for publication. Guang-ping Ruan, Xing-hua Pan, Jie He, Xiang-qing Zhu and Rong-qing Pang were involved in drafting the manuscript or revising it critically for important intellectual content. All authors read and approved the final manuscript.

\section{Funding statement}

This work was supported by grants from Yunnan Science and Technology Plan Project Major Science and Technology Project (2018ZF007), the Yunnan Province Applied Basic Research Program Key Project (2018FA041, 202101AS0 70039), the General Project of the Yunnan Applied Basic Research Program (202101AT070212), the National Natural 
Tree Shrew Umbilical Cord Mesenchymal Stem Cells Labeled with the Dark Red Fluorescent Dye DIR and Small Animal Live...

Science Foundation (31970515) and the $920^{\text {th }}$ Hospital of the PLA Joint Logistics Support Force In-hospital Technology Plan (2019YGB17, 2019YGA05).

\section{REFERENCES}

Bansal, K., Singh, C.K., Sandhu, B.S., Sood, N.K. and Dandale, M. (2014). Antemortem diagnosis of rabies from skin by taqman real time PCR. Indian Journal of Animal Research. 48: 597.

Berninger, M.T., Mohajerani, P., Kimm, M., Masius, S., Ma, X., Wildgruber, M., Haller, B., Anton, M., Imhoff, A.B., Ntziachristos, V., Henning, T.D. and Meier, R. (2017). Fluorescence molecular tomography of DiR-labeled mesenchymal stem cell implants for osteochondral defect repair in rabbit knees. Eur. Radiol. 27: 1105-1113.

Berninger, M.T., Mohajerani, P., Wildgruber, M., Beziere, N., Kimm, M.A., Ma, X., Haller, B., Fleming, M.J., Vogt, S., Anton, M., Imhoff, A.B., Ntziachristos, V., Meier, R. and Henning, T.D. (2017). Detection of intramyocardially injected DIRlabeled mesenchymal stem cells by optical and optoacoustic tomography. Photoacoustics. 6: 37-47.

Chen, B., Qin, M.C., Huang, J.L., Wu, D.P., Guo, E.C., Liu, Z.P., Xu, Z.H., Guo, X.X. and Zhong, Z.G. (2017). Preliminary establishment of integration of Alzheimer's disease and blood stasis syndrome tree shrew model and evaluation of intervention of Panax notoginseng saponins. Zhongguo Zhong Yao Za Zhi. 42: 1175-1182.

Cui, Y., Ma, S., Zhang, C., Cao, W., Liu, M., Li, D., Lv, P., Xing, Q., Qu, R., Yao, N., Yang, B. and Guan, F. (2017). Human umbilical cord mesenchymal stem cells transplantation improves cognitive function in Alzheimer's disease mice by decreasing oxidative stress and promoting hippocampal neurogenesis. Behav. Brain Res. 320: 291-301.

Dhanju, C.K., Toor, G. and Cheema, R.S. (2009). Effect of seminal plasma on quantily and qualily of proteins leaked during heparin -induced in vitro capacitation of bull sperm. Indian Journal of Animal Research. 43: 136-138.
Fahmy, S.R., Soliman, A.M., El Ansary, M., Elhamid, S.A. and Mohsen, H. (2017). Therapeutic efficacy of human umbilical cord mesenchymal stem cells transplantation against renal ischemia/reperfusion injury in rats. Tissue Cell. 49: 369-375.

Feng, Y., Feng, Y.M., Lu, C., Han, Y., Liu, L., Sun, X., Dai, J. and Xia, X. (2017). Tree shrew, a potential animal model for hepatitis $\mathrm{C}$, supports the infection and replication of HCV in vitro and in vivo. J. Gen. Virol. 98: 2069-2078.

Jiang, L.P., Shen, Q.S., Yang, C.P. and Chen, Y.B. (2017). Establishment of basal cell carcinoma animal model in Chinese tree shrew (Tupaia belangeri chinensis). Zool Res. 38: 180190.

Qiao, S.M., Wang, Y., Song, X.L. and Yue, Y.N. (2017). Research Advance on Role of Umbilical Cord Mesenchymal Stem Cell in Hematopoietic Stem Cells Transplantation-Review. Zhongguo Shi Yan Xue Ye Xue Za Zhi. 25: 1847-1850.

Rahman, S. and Gupta, J. (2015). Knowledge and adoption level of improved dairy farming practices of shg members and nonmembers in Kamrup district of Assam, India. Indian Journal of Animal Research. 49(2): 234-240.

Tong, Y., Hao, J., Tu, Q., Yu, H., Yan, L., Li, Y., Lv, L., Wang, F., lavarone, A. and Zhao, X. (2017). A tree shrew glioblastoma model recapitulates features of human glioblastoma. Oncotarget. 8: 17897-17907.

Wang, D., Niu, L., Feng, X., Yuan, X., Zhao, S., Zhang, H., Liang, J., Zhao, C., Wang, H., Hua, B. and Sun, L. (2017). Longterm safety of umbilical cord mesenchymal stem cells transplantation for systemic lupus erythematosus: A 6year follow-up study. Clin. Exp. Med. 17: 333-340.

Wu, D., Zou, S., Chen, H., Li, X., Xu, Y., Zuo, Q., Pan, Y., Jiang, S.W., Huang, H. and Sun, L. (2017). Transplantation routes affect the efficacy of human umbilical cord mesenchymal stem cells in a rat GDM model. Clin Chim Acta. 475: 137-146.

Xiao, J., Liu, R. and Chen, C.S. (2017). Tree shrew (Tupaia belangerı) as a novel laboratory disease animal model. Zool Res. 38: 127-137. 